\title{
An Evolutionary Approach for Object-Based Image Reconstruction Using Learnt Priors ${ }^{\star}$
}

\author{
Péter Balázs and Mihály Gara \\ Department of Image Processing and Computer Graphics, \\ University of Szeged, \\ Árpád tér 2., H-6720, Szeged, Hungary \\ \{pbalazs,gara\}@inf .u-szeged.hu
}

\begin{abstract}
In this paper we present a novel algorithm for reconstructing binary images containing objects which can be described by some parameters. In particular, we investigate the problem of reconstructing binary images representing disks from four projections. We develop a genetic algorithm for this and similar problems. We also discuss how prior information on the number of disks can be incorporated into the reconstruction in order to obtain more accurate images. In addition, we present a method to exploit such kind of knowledge from the projections themselves. Experiments on artificial data are also conducted.
\end{abstract}

\section{Introduction}

The aim of Computerized Tomography (CT) is to obtain information about the interior of objects without damaging or destroying them. Methods of CT (like filtered backprojection or algebraic reconstruction techniques) often require several hundreds of projections to obtain an accurate reconstruction of the studied object [8]. Since the projections are usually produced by X-ray, gamma-ray, or neutron imaging, the acquisition of them can be expensive, time-consuming or can (partially or fully) damage the examined object. Thus, in many applications it is impossible to apply reconstruction methods of CT with good accuracy. In those cases there is still a hope to get a satisfactory reconstruction by using Discrete Tomography (DT) 677. In DT one assumes that the image to be reconstructed contains just a few grey-intensity values that are known beforehand. This extra information allows one to develop algorithms which reconstruct the image from just few (usually not more than four) projections.

When the image to be reconstructed is binary we speak of Binary Tomography (BT) which has its main applications in angiography, electron microscopy, and non-destructive testing. BT is a relatively new field of research, and for a large variety of images the reconstruction problem is still not satisfactorily solved. In this paper we present a new approach for reconstructing binary images representing disks from four projections. The method is more general in the sense that it can be adopted to similar reconstruction tasks as well. The

\footnotetext{
^ This work was supported by OTKA grant T048476.
} 
paper is structured as follows. In Sect. 2 we give the preliminaries. In Sect. 3 we outline an object-based genetic reconstruction algorithm. The algorithm can use prior knowledge to grade up the reconstruction. Section 4 describes a method to collect such information when it is not explicitly given. In Sect. 5] we present experimental results. Finally, Sect. [6 is for the conclusion.

\section{Preliminaries}

The reconstruction of 3D binary objects is usually done slice-by-slice, i.e, by integrating together the reconstructions of $2 \mathrm{D}$ slices of the object. Such a $2 \mathrm{D}$ binary slice can be represented by a $2 \mathrm{D}$ binary function $f(x, y)$. The Radon transformation $\mathcal{R} f$ of $f$ is then defined by

$$
[\mathcal{R} f](s, \vartheta)=\int_{-\infty}^{\infty} f(x, y) d u,
$$

where $s$ and $u$ denote the variables of the coordinate system obtained by a rotation of angle $\vartheta$. For a fixed angle $\vartheta$ we call $\mathcal{R} f$ as the projection of $f$ defined by the angle $\vartheta$ (see Fig. 1). The reconstruction problem can be stated mathematically as follows. Given the functions $g\left(s, \vartheta_{1}\right), \ldots, g\left(s, \vartheta_{n}\right)$ (where $n$ is a positive integer) find a function $f$ such that

$$
[\mathcal{R} f]\left(s, \vartheta_{i}\right)=g\left(s, \vartheta_{i}\right) \quad(i=1, \ldots, n) .
$$

\section{An Object-Based Genetic Reconstruction Algorithm}

\subsection{Reconstruction with Optimization}

In this work we concentrate on the reconstruction of binary images representing disjoint disks inside a ring (see again Fig. 1). Such images were introduced for testing the effectiveness of reconstruction algorithms developed for neutron tomography 91011. For the reconstruction we will use just four projections. Our aim is to find a function $f$ that satisfies (2) with the given angles $\vartheta_{1}=0^{\circ}$, $\vartheta_{2}=45^{\circ}, \vartheta_{3}=90^{\circ}$, and $\vartheta_{4}=135^{\circ}$. In practice, instead of finding the exact function $f$, we are usually satisfied with a good approximation of it. On the other
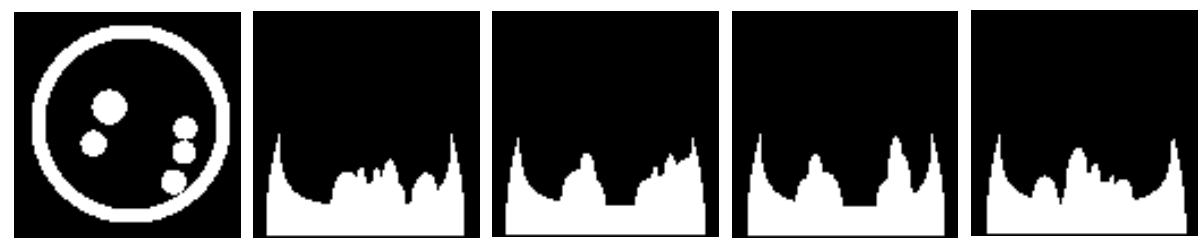

Fig. 1. A binary image and its projections defined by the angle $\vartheta=0^{\circ}, \vartheta=45^{\circ}$, $\vartheta=90^{\circ}$, and $\vartheta=135^{\circ}$ (from left to right, respectively) 
hand - especially if the number of projections is small - there can be several different functions which (approximately) satisfy (2). Fortunately, with additional knowledge of the image to be reconstructed some of them can be eliminated, which might yield that the reconstructed image will be close to the original one. For this purpose we rewrite the reconstruction task as an optimization problem where the aim is to find the minimum of the objective functional

$$
\Phi(f)=\lambda_{1} \cdot \sum_{i=1}^{4}\left\|\mathcal{R} f\left(s, \vartheta_{i}\right)-g\left(s, \vartheta_{i}\right)\right\|+\lambda_{2} \cdot \varphi\left(c_{f}, c\right) .
$$

The first term in the right hand side of (3) guarantees that the projections of the reconstructed image will be close to the prescribed ones. In the second term we can keep control over the number of disks in the image to be reconstructed. We will use this prior information to obtain more accurate reconstructions. Here, $c_{f}$ is the number of disks in the image $f$. Finally, $\lambda_{1}$ and $\lambda_{2}$ are suitably chosen scaling constants. With the aid of them we can also express whether the projections or the prior information is more reliable.

In DT (3) is usually solved by simulated annealing (SA) [12]. In [9] two different approaches were presented to reconstruct binary images representing disks inside a ring with SA. The first one is a pixel-based method where in each iteration a single pixel value is inverted to obtain a new proposed solution. Although this method can be applied in more general (i.e., also in the case when the image does not represent disks), it has some serious drawbacks: it is quite sensitive to noise, it can not exploit geometrical information of the image to be reconstructed, and it needs 10-16 projections for an accurate reconstruction. The other method of [9] is a parameter-based one in which the image is represented by the centers and radii of the disks, and the aim is to find the proper setting of these parameters. This algorithm is less sensitive to noise, easy to extend to direct $3 \mathrm{D}$ reconstruction, but its accuracy decreases drastically as the complexity of the image (i.e. the number of disks in it) increases. Furthermore, the number of disks should be given before the reconstruction. In this paper we design an algorithm that can benefit the advantages of both reconstruction methods. However, instead of using SA to find an approximately good solution, we will describe an evolutionary approach. Evolutionary computation 22 proved to be successful in many large-scale optimization tasks. Unfortunately, the pixel-based representation of the image makes evolutionary algorithms difficult to use in binary image reconstruction. Nevertheless, some efforts have already been done to overcome this problem in tricky ways 3514. Our idea is a more natural one, we will use a parameter-based representation of the image.

\subsection{Entity Representation}

We assume that there exists a ring which center coincides the center of the image, and there are some disjoint disks inside this ring (the ring and each of the disks are disjoint as well) (see, e.g., Fig. 1). The outer ring can be represented as the difference of two disks, and therefore the whole image can be described by a 
list of triplets $\left\langle\left(x_{1}, y_{1}, r_{1}\right), \ldots,\left(x_{n}, y_{n}, r_{n}\right)\right\rangle$ where $n \geq 3$. Here, $\left(x_{i}, y_{i}\right)$ and $r_{i}$ $(i=1, \ldots, n)$ denote the center and the radius of the $i^{\text {th }}$ disk, respectively (the bottom-left corner of the image is $(0,0))$. Since the first two elements of the list stand for the outer ring, $x_{1}=x_{2}, y_{1}=y_{2}$, and $r_{1}>r_{2}$ do always hold. Moreover, the point $\left(x_{1}, y_{1}\right)$ is the center of the image.

The evolutionary algorithm seeks for the optimum by a population of entities. Each entity is a suggestion for the optimum, and its fitness is simply measured by the formula of (3) (smaller values belong to better solutions). The entities of the actual population are modified with the mutation and crossover operators. These are described in the followings in more detail.

\subsection{Crossover}

Crossover is controlled by a global probability parameter $p_{c}$. During the crossover each entity $e$ is assigned a uniform randomly chosen number $p_{e} \in[0,1]$. If $p_{e}<p_{c}$ then the entity is subject to crossover. In this case we randomly choose an other entity $e^{\prime}$ of the population and try to cross it with $e$. Suppose that $e$ and $e^{\prime}$ are described by the lists $\left\langle\left(x_{1}, y_{1}, r_{1}\right), \ldots,\left(x_{n}, y_{n}, r_{n}\right)\right\rangle$ and $\left\langle\left(x_{1}^{\prime}, y_{1}^{\prime}, r_{1}^{\prime}\right), \ldots,\left(x_{k}^{\prime}, y_{k}^{\prime}, r_{k}^{\prime}\right)\right\rangle$, respectively $\left(e\right.$ and $e^{\prime}$ can have different number of disks, i.e., $k$ is not necessarily equal to $n)$. Then the two offsprings are presented by $\left\langle\left(x_{1}, y_{1}, r_{1}\right), \ldots,\left(x_{t}, y_{t}, r_{t}\right),\left(x_{s+1}^{\prime}, y_{s+1}^{\prime}, r_{s+1}^{\prime}\right), \ldots,\left(x_{k}^{\prime}, y_{k}^{\prime}, r_{k}^{\prime}\right)\right\rangle$ and $\left\langle\left(x_{1}^{\prime}, y_{1}^{\prime}, r_{1}^{\prime}\right), \ldots,\left(x_{s}^{\prime}, y_{s}^{\prime}, r_{s}^{\prime}\right),\left(x_{t+1}, y_{t+1}, r_{t+1}\right), \ldots,\left(x_{n}, y_{n}, r_{n}\right)\right\rangle$ where $3 \leq t \leq n$ and $3 \leq s \leq k$ are chosen from uniform random distributions. As special cases an offspring can inherit all or none of the innner disks of one of its parents (the method guarantees that the outer rings in both parent images are kept). A crossover is valid if the ring and all of the disks are pairwisely disjoint in the image. Though, in some cases it can happen that both offsprings are invalid. In this case we repeat to choose $s$ and $t$ randomly until at least one of the offsprings is valid or we reach the maximal number of allowed attempts $a_{c}$. Figure2 shows an example for the crossover. The list of the two parents are $\langle(50,50,40.01)$, $(50,50,36.16),(41.29,27.46,8.27),(65.12,47.3,5.65),(54.69,55.8,5),(56.56$, $73.38,5.04),(46.49,67.41,5)\rangle$ and $\langle(50,50,45.6),(50,50,36.14),(40.33,24.74$, $7.51),(24.17,54.79,7.59),(74.35,46.37,10.08)\rangle$. The offsprings are $\langle(50,50$, $45.6),(50,50,36.14)$, (40.33, 24.74, 7.51), (24.17, 54.79, 7.59), (54.69, 55.8, 5), $(56.56,73.38,5.04),(46.49,67.41,5)\rangle$ and $\langle(50,50,40.01),(50,50,36.16),(41.29$ $27.46,8.27),(65.12,47.3,5.65),(74.35,46.37,10.08)\rangle$.

\subsection{Mutation}

During the mutation an entity can change in three different ways:

(1) the number of disks increases/decreases by 1 ,

(2) the radius of a disk changes by at most 5 units, or

(3) the center of a disk moves inside a circle having a radius of 5 units.

For each type of the above mutations we set global probability thresholds, $p_{m_{1}}$, $p_{m_{2}}$, and $p_{m_{3}}$, respectively, which have the same roles as $p_{c}$ has for crossover. For 

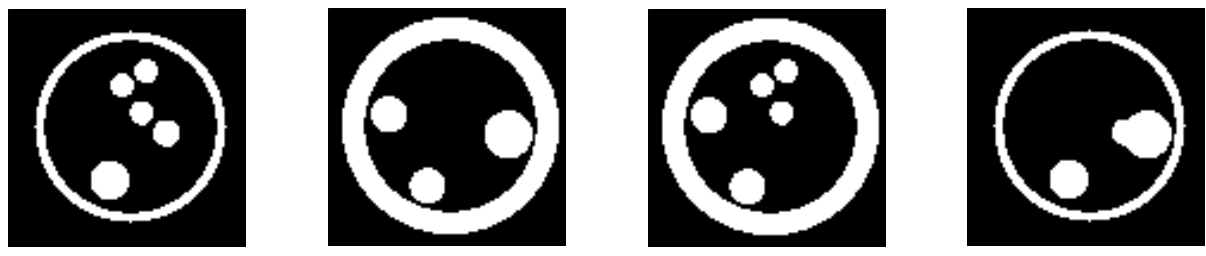

Fig. 2. An example for crossover. The images are the two parents, a valid, and an invalid offspring (from left to right).
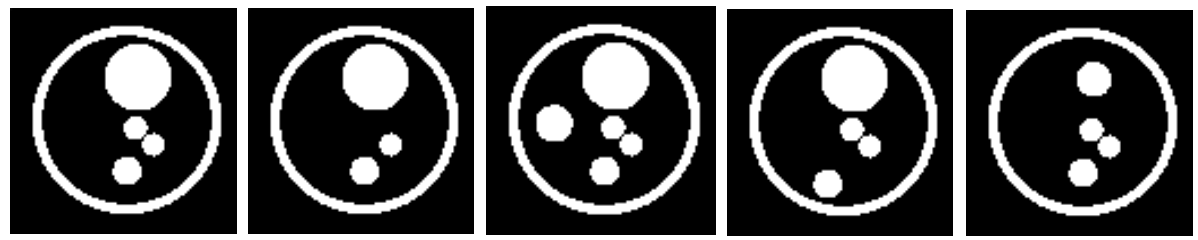

Fig. 3. Examples for mutation. From left to right: original image, decreasing and increasing the number of disks, moving the center of a disk, and resizing a disk.

the first type of mutation the number of disks is increased and decreased with equal $0.5-0.5$ probability. If the number of disks is increased then we add a new element to the end of the list. If this newly added element intersects any element of the list (except itself) then we do a new attempt. We repeat this method until we succeed or the maximal number of attempts $a_{m}$ is reached. When the number of disks should be decreased then we simply delete one element of the list (which cannot be among the first two elements since the ring should be unchanged).

In the case when the radius of a disks had to be changed then this disk is randomly chosen from the list and we modify its radius by a randomly chosen value from the interval $[-5,5]$. The disk to modify can be one of the disks describing the ring, as well. Finally, if we move the center of a disk then it is done again with uniform random distribution in a given interval. In this case the ring can not be subject to change. In the last two types of mutation we do not take another attempts if the mutated entity is not valid. Figure 3 shows examples of the several mutation types.

\subsection{Selection}

During the genetic process the population consists of a fixed number (say $\gamma$ ) of entities, and only entities with the best fitness values will survive to the next generation. In each iteration we first apply the crossover operator with which we obtain $\mu_{1}$ (valid) offsprings. In this stage all the parents and offsprings are present in the population. With the aid of the mutation operators we obtain $\mu_{2}$ new entities from the $\gamma+\mu_{1}$ entities and we also add them to the population. Finally, from the $\gamma+\mu_{1}+\mu_{2}$ number of entities we only keep $\gamma$ having the best fitness values and they will form the next generation. 


\section{Guessing the Number of Disks}

Our final aim is to design a reconstruction algorithm that can cleverly use the knowledge of the number of disks present in the image. The method developed in 9] assumes that this information is available beforehand. In contrary, we try to exploit it from the projections themselves, thus, making or method more flexible, and more widely applicable. Our preliminary investigations showed that decision trees can help to gain structural information from the projections of a binary image [1. Therefore we again used C4.5 tree classifiers for this task [13].

With the aid of the generator algorithm of DIRECT [4] we generated 11001100 images having $1,2, \ldots, 10$ disks inside the outer ring. All of them were of size of $100 \times 100$ and the number of projections were 100 from each directions. We used 1000 images from each set to train the tree, and the remaining 100 to test the accuracy of the classification. Our decision variables were the number of local optima and their values in all four projection vectors. In this way we had $4(1+6)=28$ variables for each training example and we classified those examples into 10 classes (depending on the number of disks in the image from which the projections arose). If the number of local maxima was less than 6 then we simply set the corresponding decision variable to be 0 , if this number was greater than six, then the remaining values were omitted. Table 1 shows the results of classification of the decision tree on the test data set. Although the tree built during the learning was not able to predict the exact number of disks with good accuracy (except if the image contained just a very few disks) its classification can be regarded quite accurate if we allow an error of 1 or 2 disks. This observation turns out to be useful to add information on the number of disks into the fitness function of our genetic algorithm. We set the term $\varphi\left(c_{f}, c\right)$ in the fitness function in the following way

$$
\varphi\left(c_{f}, c\right)=1-\frac{t_{c_{f}, c}}{\sum_{i=1}^{10} t_{i, c}}
$$

where $c$ is the class given by the decision tree by using the projections, and $t_{i j}$ denotes the element of Table 1 in the $i$-th row and the $j$-th column. For example,

Table 1. Predicting the number of disks by a decision tree from the projection data

\begin{tabular}{|c|c|c|c|c|c|c|c|c|c|c|}
\hline (a) & (b) & (c) & $\overline{(d)}$ & (e) & $(\mathrm{f})$ & $(\mathrm{g})$ & $\overline{(h)}$ & (i) & $(\mathrm{j})$ & $<$-classified as \\
\hline 100 & & & & & & & & & & (a): class 1 \\
\hline & 92 & 8 & & & & & & & & (b): class 2 \\
\hline & 8 & 75 & 16 & 1 & & & & & & (c): class 3 \\
\hline & & 23 & 49 & 23 & 3 & 2 & & & & (d): class 4 \\
\hline & & & 2 & 21 & 45 & 22 & 5 & 5 & & (e): class 5 \\
\hline & & & 6 & 22 & 35 & 24 & 7 & 5 & 1 & (f): class 6 \\
\hline & & & & 8 & 25 & 26 & 22 & 14 & 5 & (g): class 7 \\
\hline & & & & 3 & 12 & 16 & 30 & 23 & 16 & (h): class 8 \\
\hline & & & & & 5 & 15 & 18 & 25 & 37 & (i): class 9 \\
\hline & & & & & & 7 & 20 & 29 & 44 & (j): class 10 \\
\hline
\end{tabular}


if on the basis of the projection vectors the decision tree predicts that the image to be reconstructed has five inner disks (class (e)) then for an arbitrary image $f$ $\varphi\left(c_{f}, 5\right)$ is equal to $1.0,1.0,0.9871,0.7051,0.7307,0.7179,0.8974,0.9615,1.0$, and 1.0 for $c_{f}=1, \ldots, 10$, respectively.

\section{Experimental Results}

In order to test the efficacy of our method we conducted the following experiment. We designed 10 test images with increasing structural complexity having $1,2, \ldots, 10$ disks inside the ring. We tried to reconstruct each image 10 times by our approach with no information about the number of disks, 10 times with the information defined by (44), and finally 10 times when we assumed that the number of disks is known in advance (by setting $\varphi$ to be 0.0 if the reconstructed image had the same number of disks as it was expected and 1.0 otherwise).

The initial population consisted of 200-200 entities from the classes 3 to 9 (i.e. we used $\gamma=1400$ ). For the random generation of the entities we again used the algorithm of DIRECT [4. The threshold parameters for the operators were set to $p_{c}=0.05, p_{m_{1}}=0.05$, and $p_{m_{2}}=p_{m_{3}}=0.25$. The maximal number of attempts were $a_{c}=50$ for the crossover and $a_{m}=1000$ for the mutation of the first type. We found the best results with setting $\lambda_{1}=0.000025$ and $\lambda_{2}=0.015$. We set the reconstruction process to terminate after 3000 generations. Figure 4 represents the best reconstruction results achieved by the three methods.

To the numerical evaluation of the accuracy of our method we used the relative mean error (RME) that was defined in [9] as

$$
R M E=\frac{\sum_{i}\left|f_{i}^{o}-f_{i}^{r}\right|}{\sum_{i} f_{i}^{o}} \cdot 100 \%
$$

where $f_{i}^{o}$ and $f_{i}^{r}$ denote the $i^{\text {th }}$ pixel of the original and the reconstructed image, respectively. Thus, the smaller the RME value is, the better the reconstruction is. The numerical results are given in Table 2 and - for the sake of transparency - they are also shown on a graph (see Fig. 5). On the basis of this experiment we can deduce that all three variants of our method perform quite well for simple images (say, for images having less than 5-6 disks), and give results that can be suitable for practical applications, as well. Just for a comparison, the best reconstruction obtained by our method using four projections for the test image having 4 inner disks gives an RME of 1.95\%, while the pixel-based method of [9] on an image having the same complexity yields an RME of $12.57 \%$ by using eight (!) projections (cf. 9] for more sophisticated comparisons). For more complex images the reconstruction becomes more inaccurate. However, the best results are usually achieved by the decision tree approach, and it still gives images of relatively good quality. Regarding the reconstruction time we found that it is about 10 minutes for images having few (say, 1-3 inner disks), 30 minutes if there are more than 3 disks, and 1 hour for images having 8-10 disks (on an Intel Celeron $2.8 \mathrm{GHz}$ processor with $1.5 \mathrm{~GB}$ of memory). 

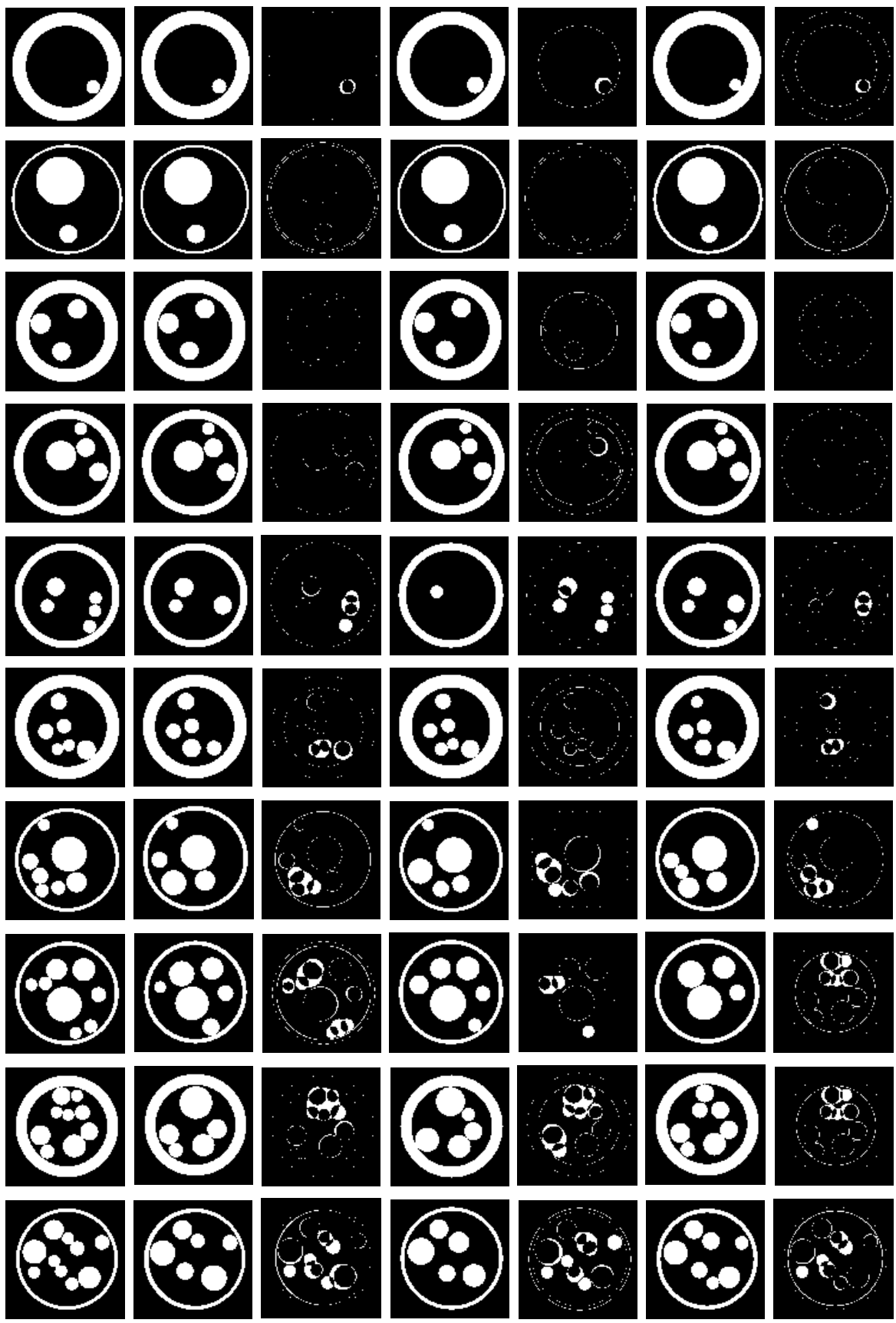

Fig. 4. Reconstruction with the genetic algorithm. From left to right: Original image, reconstruction with no prior information, the difference image, reconstruction with fix prior information, the difference image, and reconstruction with the decision tree approach and the difference image. 
Table 2. RME (rounded to two digits) of the best out of 10 reconstructions as it depends on the number of inner disks (first row) with no prior information (second row), fix (third row), and learnt prior information (fourth row). In the latter case the number of disks predicted by the decision tree is given in the fifth row.

\begin{tabular}{|c|c|c|c|c|c|c|c|c|c|}
\hline 1 & 2 & 3 & 4 & 5 & 6 & 7 & 8 & 9 & 10 \\
\hline \hline 1.92 & 8.66 & 0.78 & 2.29 & 13.86 & 7.72 & 19.63 & 29.00 & 12.06 & 33.51 \\
\hline 3.60 & 4.50 & 3.01 & 7.16 & 4.27 & 5.51 & 22.31 & 11.20 & 17.05 & 39.52 \\
\hline 4.75 & 11.32 & 1.22 & 1.95 & 8.08 & 6.15 & 17.98 & 26.42 & 12.09 & 28.48 \\
\hline 1 & 2 & 3 & 5 & 5 & 8 & 5 & 10 & 7 & 10 \\
\hline
\end{tabular}

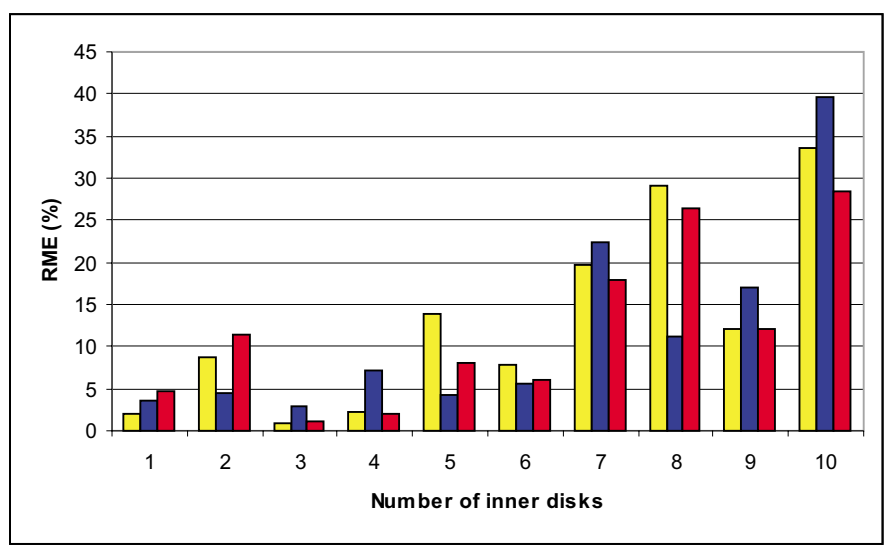

Fig. 5. Relative mean error of the best out of 10 reconstructions with no prior information (left column), fix priors (middle column), and learnt priors (right column)

\section{Conclusion and Further Work}

We have developed an evolutionary algorithm for object-based binary image reconstruction which can handle prior knowledge also in the case when it is not explicitly given. We used decision trees for learning prior information, but the framework is easy to adapt to use other classifiers, as well. Experimental results show that each variant of our algorithm is promising, but some work still have to be done. We found that the repetition of the mutation and crossover operators until a valid offspring is generated can take quite a long time - especially if there are many disks in the image. Our future aim is to develop faster mutation and crossover operators. In our further work we also want to tune the parameters of our algorithm to achieve more accurate reconstructions. This includes finding more sophisticated attributes that can be used in the decision tree for describing the number of disks present in the image. The study of noise-sensitivity and possible 3D extensions of our method form also parts of our further research. 


\section{References}

1. Balázs, P., Gara, M.: Decision trees in binary tomography for supporting the reconstruction of $h v$-convex connected images. In: Blanc-Talon, J., Bourennane, S., Philips, W., Popescu, D., Scheunders, P. (eds.) ACIVS 2008. LNCS, vol. 5259, pp. 433-443. Springer, Heidelberg (2008)

2. Bäck, T., Fogel, D.B., Michalewicz, T. (eds.): Evolutionary Computation 1. Institute of Physics Publishing, Bristol (2000)

3. Batenburg, K.J.: An evolutionary algorithm for discrete tomography. Disc. Appl. Math. 151, 36-54 (2005)

4. DIRECT - DIscrete REConstruction Techniques. A toolkit for testing and comparing $2 \mathrm{D} / 3 \mathrm{D}$ reconstruction methods of discrete tomography, http://www.inf.u-szeged.hu/ direct

5. Di Gesù, V., Lo Bosco, G., Millonzi, F., Valenti, C.: A memetic algorithm for binary image reconstruction. In: Brimkov, V.E., Barneva, R.P., Hauptman, H.A. (eds.) IWCIA 2008. LNCS, vol. 4958, pp. 384-395. Springer, Heidelberg (2008)

6. Herman, G.T., Kuba, A. (eds.): Discrete Tomography: Foundations, Algorithms and Applications. Birkhäuser, Boston (1999)

7. Herman, G.T., Kuba, A. (eds.): Advances in Discrete Tomography and its Applications. Birkhäuser, Boston (2007)

8. Kak, A.C., Slaney, M.: Principles of Computerized Tomographic Imaging. IEEE Press, New York (1988)

9. Kiss, Z., Rodek, L., Kuba, A.: Image reconstruction and correction methods in neutron and x-ray tomography. Acta Cybernetica 17, 557-587 (2006)

10. Kiss, Z., Rodek, L., Nagy, A., Kuba, A., Balaskó, M.: Reconstruction of pixelbased and geometric objects by discrete tomography. Simulation and physical experiments. Elec. Notes in Discrete Math. 20, 475-491 (2005)

11. Kuba, A., Rodek, L., Kiss, Z., Ruskó, L., Nagy, A., Balaskó, M.: Discrete tomography in neutron radiography. Nuclear Instr. Methods in Phys. Research A 542, 376-382 (2005)

12. Metropolis, N., Rosenbluth, A., Rosenbluth, M., Teller, E.: Equation of state calculation by fast computing machines. J. Chem. Phys. 21, 1087-1092 (1953)

13. Quinlan, J.R.: C4.5: Programs for Machine Learning. Morgan Kaufmann, San Francisco (1993)

14. Valenti, C.: A genetic algorithm for discrete tomography reconstruction. Genet. Program Evolvable Mach. 9, 85-96 (2008) 